\title{
Recent results on hot topics from Belle
}

\author{
Gagan B. Mohanty \\ Tata Institute of Fundamental Research, Homi Bhabha Road, \\ Colaba, Mumbai 400 005, INDIA
}

\begin{abstract}
We report a sample of recent and topical physics results based on the data recorded with the Belle detector at the KEK $B$-factory in Japan.

Keywords: Charm mixing, $C P$ violation, rare $B$ decays, exotic meson

\section{Introduction}

The Belle experiment is a multitasking magnetic spectrometer that operated for more than a decade at the KEKB asymmetric-energy $e^{+} e^{-}$collider. Before closing down in June 2010 to make way for its upgrade (Belle II), it has succeeded in collecting a world-record sample of data over $1 \mathrm{ab}^{-1}$ near various $\Upsilon(n S)$ resonances. We present herein a sample of recent interesting results from Belle based on its full statistics.
\end{abstract}

\section{Charm mixing and $C P$ violation}

Under weak interaction a flavored neutral meson can be represented as a two-state quantum system, leading to possible transition between the two states. The phenomenon going by the name neutral meson mixing is intimately related to the difference between the flavor and mass eigenstates of the meson-antimeson system. Such mixing is already well established for $K^{0}, B^{0}$ and $B_{s}^{0}$ mesons. The observed mixing rates are consistent with the standard model (SM) predictions that depend on the Cabibbo-Kobayashi-Maskawa (CKM) matrix [1] elements, appearing in the short-distance box diagrams for mixing. For $D^{0}$ mesons the box diagrams are, however, both Cabibbo and GIM [2] suppressed giving rise to a small contribution. Consequently, $D^{0}-\bar{D}^{0}$ mixing is dominated by long-distance processes that are difficult to calculate. Theoretical estimates for the mixing parameters $x \equiv \Delta m / \Gamma$ and 
$y \equiv \Delta \Gamma / 2 \Gamma$ range over two to three orders of magnitude [3]. Here, $\Delta m$ and $\Delta \Gamma$ are the mass and decay width differences between the two $D$ mass eigenstates, and $\Gamma$ is their average decay width.

Violation of charge-parity $(C P)$ symmetry in the charm sector provides an interesting test for new physics (NP) as the SM predicts a very small asymmetry owing to: a) large Cabibbo and GIM suppression, similar to mixing, and b) a lack of large hierarchy in the down-type quark masses. Among various $D$ decay modes the singly Cabibbo-suppressed (SCS) decays constitute the most promising candidate to probe $C P$ violation [4] with typical SM values are of the order of $10^{-3}$. With respect to such percentage effects one needs a good control on the theory prediction, something that is in general lacking in the charm sector due to long-distance effects. Furthermore, with $D^{0}-\bar{D}^{0}$ mixing being firmly established (see below) one would like to explore possible $C P$ violation in mixing or due to interference between mixing and decay amplitudes.

\section{Mixing in $D \rightarrow K \pi$ decays}

We search for $D^{0}-\bar{D}^{0}$ mixing in $D \rightarrow K \pi$ decays [5] by taking a ratio of the time-dependent rate of the wrong-sign (WS) decay $D^{0} \rightarrow K^{+} \pi^{-}$to that of the right-sign (RS) decay $D^{0} \rightarrow K^{-} \pi^{+}$. The RS and WS processes are identified via the decay chain $D^{\star+} \rightarrow D^{0}\left(K^{\mp} \pi^{ \pm}\right) \pi_{s}^{+}$, where one compares the charge of the slow pion $\pi_{s}$ with that of the pion arising from the $D$ decay. If the two are the same, it would be a RS decay; else, a WS decay. The RS amplitude is dominated by a Cabibbo-favored ( $\mathrm{CF}$ ) decay $D^{0} \rightarrow K^{-} \pi^{+}$with a negligible contribution from $D^{0}-\bar{D}^{0}$ mixing followed by a doubly Cabibbosuppressed (DCS) decay $\bar{D}^{0} \rightarrow K^{-} \pi^{+}$. In contrast, for the WS case the two contributing amplitudes, from the DCS decay $D^{0} \rightarrow K^{+} \pi^{-}$and $D^{0}-\bar{D}^{0}$ mixing followed by the CF decay $\bar{D}^{0} \rightarrow K^{+} \pi^{-}$, are of similar magnitude. The time-dependent ratio of WS to RS decay rates is thus given by

$$
R(t / \tau) \equiv \frac{\Gamma_{\mathrm{WS}}(t / \tau)}{\Gamma_{\mathrm{RS}}(t / \tau)} \approx R_{D}+\sqrt{R_{D}} y^{\prime} \frac{t}{\tau}+\frac{x^{\prime 2}+y^{\prime 2}}{4}\left(\frac{t}{\tau}\right)^{2}
$$

to the second order in mixing parameters. Here, $t$ is the proper decay time, $\tau$ is the average $D^{0}$ lifetime, $R_{D}$ is the ratio of DCS to CF decay rates, $x^{\prime}=x \cos \delta+y \sin \delta$, and $y^{\prime}=-x \sin \delta+y \cos \delta$ are the "rotated" mixing parameters with $\delta$ being the strong phase difference between the DCS and CF decay amplitudes. 
Table 1: Results of the time-dependent fit to $R(t / \tau)$, where DOF denotes the number of degrees of freedom. The uncertainties are statistical and systematic combined.

\begin{tabular}{lccc}
\hline \hline Test hypothesis & $\chi^{2} /$ DOF & Parameter & Fit result $\left(10^{-3}\right)$ \\
\hline Mixing & $4.2 / 7$ & $R_{D}$ & $3.53 \pm 0.13$ \\
& & $y^{\prime}$ & $4.6 \pm 3.4$ \\
& & $x^{\prime 2}$ & $0.09 \pm 0.22$ \\
\hline No mixing & $33.5 / 9$ & $R_{D}$ & $3.864 \pm 0.059$ \\
\hline \hline
\end{tabular}

Based on a $976 \mathrm{fb}^{-1}$ of data sample, we select 2, 980,710 $\pm 1885 \mathrm{RS}$ and $11,478 \pm 177$ WS decay candidates. The proper decay time of these decay candidates is calculated as $t=m_{D^{0}} \vec{L} \cdot \vec{p} /|\vec{p}|^{2}$, where $\vec{L}$ is a vector joining the $D^{0}$ production and decay vertices, $\vec{p}$ is the $D^{0}$ momentum, and $m_{D^{0}}$ is the nominal $D^{0}$ mass [6]. Due care has been taken in incorporating detector resolution effects to the measured $t$ distribution. We fit the obtained timedependent decay rate ratios according to the expression in Eq. (11). Two hypotheses, with and without $D^{0}-\bar{D}^{0}$ mixing, are tested and the corresponding results are given in Table 1. The $\chi^{2}$ difference between the two is 29.3 for 2 degrees of freedom, corresponding to a probability of $4.3 \times 10^{-7}$. This means, the no-mixing hypothesis is excluded at a level of 5.1 standard deviations $(\sigma)$. Our results constitute the first observation of $D^{0}-\bar{D}^{0}$ mixing from a single $e^{+} e^{-}$collider experiment, and are in agreement with those from experiments at the hadron machine [7].

\section{Mixing and $C P$ violation in $D \rightarrow K_{S}^{0} \pi^{+} \pi^{-}$}

We simultaneously probe mixing and $C P$ violation [8] by studying time dependence of the Dalitz plot in the self-conjugated decay $D^{0} \rightarrow K_{S}^{0} \pi^{+} \pi^{-}$ (denoted by $f$ below). For a given point in the Dalitz plot $\left[m_{+}^{2} \equiv m^{2}\left(K_{S}^{0} \pi^{+}\right)\right.$, $\left.m_{-}^{2} \equiv m^{2}\left(K_{S}^{0} \pi^{-}\right)\right]$, the decay amplitude is

$$
\mathcal{M}(t)=\mathcal{A}_{f}\left(m_{+}^{2}, m_{-}^{2}\right) \frac{\mathrm{e}_{1}(t)+\mathrm{e}_{2}(t)}{2}+\frac{q}{p} \mathcal{A}_{\bar{f}}\left(m_{+}^{2}, m_{-}^{2}\right) \frac{\mathrm{e}_{1}(t)-\mathrm{e}_{2}(t)}{2},
$$

where $q, p$ are the complex coefficients that relate mass to flavor eigenstates, and $\mathrm{e}_{1,2}(t)=\mathrm{e}^{-\left(i m_{1,2}+\Gamma_{1,2} / 2\right) t}$ with $m_{1,2}$ and $\Gamma_{1,2}$ being the mass and decay 
width of the mass eigenstates. The first term is the time-dependent amplitude for the (direct) decay $D^{0} \rightarrow K_{S}^{0} \pi^{+} \pi^{-}$, and the second one is the amplitude for $D^{0}-\bar{D}^{0}$ mixing followed by $\bar{D}^{0} \rightarrow K_{S}^{0} \pi^{+} \pi^{-}$. Taking the modulus squared of Eq. (2) gives the time-dependent $D^{0}$ decay rate as

$$
\begin{aligned}
|\mathcal{M}(t)|^{2}= & \frac{\mathrm{e}^{-\Gamma t}}{2}\left\{\left(\left|\mathcal{A}_{f}\right|^{2}+\left|\frac{q}{p}\right|^{2}\left|\mathcal{A}_{\bar{f}}\right|^{2}\right) \cosh (\Gamma y t)+\left(\left|\mathcal{A}_{f}\right|^{2}-\left|\frac{q}{p}\right|^{2}\left|\mathcal{A}_{\bar{f}}\right|^{2}\right)\right. \\
& \left.\cosh (\Gamma x t)+2 \mathcal{R}\left(\frac{q}{p} \mathcal{A}_{\bar{f}} A_{f}^{\star}\right) \sinh (\Gamma y t)-2 \mathcal{I}\left(\frac{q}{p} \mathcal{A}_{\bar{f}} A_{f}^{\star}\right) \sinh (\gamma x t)\right\} .
\end{aligned}
$$

The equivalent quantity in the $\bar{D}^{0}$ case also contains terms proportional to $\cosh (\Gamma x t), \cosh (\Gamma y t), \sinh (\Gamma x t)$ and $\sinh (\Gamma y t)$. Thus, by fitting the timedependent $D^{0}$ and $\bar{D}^{0}$ decay rates we can determine mixing parameters $x$ and $y$ as well as look for mixing-induced $C P$ violation by checking whether $|q / p|$ deviates from unity or $\operatorname{Arg}(q / p)$ from zero, and if so, by how much.

We use an isobar model [9] to describe the decay amplitude $\mathcal{A}_{f}\left(m_{+}^{2}, m_{-}^{2}\right)$ as $\sum_{j} a_{j} \mathrm{e}^{i \delta_{j}} A_{j}$, where $a_{j}$ and $\delta_{j}$ are the magnitude and phase of a given intermediate state $j$, and $A_{j}$ is the product of a relativistic Breit-Wigner function and Blatt-Weisskopf form factors. Thus in the Dalitz-plot fit we obtain amplitudes and phases of various intermediate states, that lead to the final state of $K_{S}^{0} \pi^{+} \pi^{-}$, in addition to mixing parameters $x$ and $y$. For no direct $C P$ violation, $\mathcal{A}_{f}\left(m_{+}^{2}, m_{-}^{2}\right)=\mathcal{A}_{\bar{f}}\left(m_{-}^{2}, m_{+}^{2}\right)$. Otherwise, one needs to separately consider $\left(a_{j}, \delta_{j}\right)$ for $D^{0}$ and $\left(\bar{a}_{j}, \bar{\delta}_{j}\right)$ for $\bar{D}^{0}$ decays.

Using a data sample of $921 \mathrm{fb}^{-1}$ recorded near the $\Upsilon(n S)(n=4,5)$ resonances with Belle, we select 1,231,731 \pm 1633 signal candidates with a purity above 95\%. We perform a time-integrated fit to the Dalitz plot distributions of these events by varying the amplitudes and phases for different intermediate states, separately for $D^{0}$ and $\bar{D}^{0}$ decays. As the two sets of parameters are found to be consistent within uncertainties, we set $\mathcal{A}_{f}\left(m_{+}^{2}, m_{-}^{2}\right)=\mathcal{A}_{\bar{f}}\left(m_{-}^{2}, m_{+}^{2}\right)$. Of course, this assumption of no direct $C P$ violation has no bearing for $C P$ violation related to mixing. Next we follow a two-step fit procedure. In the first step, we fit to a combined sample of $D^{0}$ and $\bar{D}^{0}$ with the fit observables $(x, y)$, the $D^{0}$ lifetime $\tau$, some timing resolution parameters, and isobar model parameters $\left(a_{j}, \delta_{j}\right)$. In the second step, $C P$ violation related to mixing is allowed resulting in two more fit observables: $|q / p|$ and $\phi=\operatorname{Arg}(q / p)$. Results of the above two fits are listed in Table 2. The two sets of mixing parameters obtained are pretty much identical, and they constitute a $2.5 \sigma$ evidence for $D^{0}-\bar{D}^{0}$ mixing. With $|q / p|$ and 
$\phi$ being consistent with unity and zero, respectively, there is no $C P$ violation either in $D^{0}-\bar{D}^{0}$ mixing or due to interference between mixing and decay.

Table 2: Results for mixing parameters $x$ and $y$ from the no and with $C P$ violation (CPV) case. The uncertainties are respectively statistical, experimental systematic, and the error due to amplitude model.

\begin{tabular}{lcc}
\hline \hline Fit type & Parameter & Fit result \\
\hline No CPV & $x(\%)$ & $0.56 \pm 0.19_{-0.09}^{+0.03}{ }_{-0.09}^{+0.06}$ \\
& $y(\%)$ & $0.30 \pm 0.15_{-0.05}^{+0.04}+0.03$ \\
With CPV & $x(\%)$ & $0.56 \pm 0.19_{-0.08}^{+0.04}+0.06$ \\
& $y(\%)$ & $0.30 \pm 0.15_{-0.05}^{+0.04}+0.03$ \\
& $|q / p|$ & $0.90_{-0.15}^{+0.16}+0.05+_{-0.04}^{+0.06}$ \\
& $\phi\left({ }^{\circ}\right)$ & $-6 \pm 13_{-4}^{+0.05}$ \\
\hline \hline
\end{tabular}

\section{Search for $C P$ violation in $D^{0} \rightarrow \pi^{0} \pi^{0}$}

The $D^{0} \rightarrow \pi^{0} \pi^{0}$ decay, a typical SCS one, is expected to exhibit large $C P$ violation in several NP models such as those with large chromomagnetic dipole operators [10] and the triplet model [11]. The enhanced $C P$ asymmetry value could lie anywhere between $1 \%$ and $8 \%$. Further motivation for our study came from an early measurement by LHCb [12], supported by CDF [13], suggesting a $3.5 \sigma$ effect on the difference of direct $C P$ asymmetries $\left(\triangle A_{C P}\right)$ between $D^{0} \rightarrow K^{+} K^{-}$and $D^{0} \rightarrow \pi^{+} \pi^{-}$decays. Though the current world average for $\Delta A_{C P}$ stands $2.3 \sigma$ away from zero [18], there exists a good deal of theoretical motivation [10, 11, 14] to look for $C P$ violation in $D^{0} \rightarrow \pi^{0} \pi^{0}$. Experimentally, the previous result from CLEO [15] was consistent with zero with an uncertainty of $4.8 \%$ - as big as the NP prediction.

We measure the time-integrated $C P$ asymmetry in the $D^{0} \rightarrow \pi^{0} \pi^{0}$ and $D^{0} \rightarrow K_{S}^{0} \pi^{0}$ decays [16] using $966 \mathrm{fb}^{-1}$ of data collected with Belle. In the process $D^{\star+} \rightarrow D^{0} \pi_{s}^{+}$coming from $e^{+} e^{-} \rightarrow c \bar{c}$, the charge of the lowmomentum or "slow" pion $\pi_{s}^{+}$determines the flavor of the neutral charm 
meson (whether it is a $D^{0}$ or a $\bar{D}^{0}$ ). The measured asymmetry

$$
A_{\mathrm{rec}}=\frac{N_{\mathrm{rec}}^{D^{\star+} \rightarrow D^{0} \pi_{s}^{+}}-N_{\mathrm{rec}}^{D^{\star-} \rightarrow \bar{D}^{0} \pi_{s}^{-}}}{N_{\mathrm{rec}}^{D^{\star+} \rightarrow D^{0} \pi_{s}^{+}}+N_{\mathrm{rec}}^{D^{\star-} \rightarrow \bar{D}^{0} \pi_{s}^{-}}},
$$

where $N_{\text {rec }}^{i}$ is the number of reconstructed events tagged as $i$, has three contributions: the underlying $C P$ asymmetry $A_{C P}$, the forward-backward asymmetry $\left(A_{\mathrm{FB}}\right)$ due to $\gamma-Z^{\star}$ interference in $e^{+} e^{-} \rightarrow c \bar{c}$ and higher-order QED processes, and the detection asymmetry between positively and negatively charged pions $\left(A_{\epsilon}^{\pi_{s}}\right)$. We subtract the $A_{\text {rec }}$ measured in the CF decay $D^{0} \rightarrow K^{-} \pi^{+}$("untagged") from $D^{\star+} \rightarrow D^{0} \pi_{s}^{+} ; D^{0} \rightarrow K^{-} \pi^{+}$("tagged") to estimate $A_{\epsilon}^{\pi_{s}}$. The implicit assumption here is that both $D^{\star}$ and $D$ mesons have the same $A_{\mathrm{FB}}$ value. After correcting for $A_{\epsilon}^{\pi_{s}}$, one is left with

$$
A_{\mathrm{rec}}^{\mathrm{cor}}=A_{C P}+A_{\mathrm{FB}}\left(\cos \theta^{\star}\right),
$$

where $\theta^{\star}$ is the $D^{\star+}$ polar angle in the center of mass (CM) frame. While $A_{C P}$ is independent of kinematics, $A_{\mathrm{FB}}$ is an odd function of $\cos \theta^{\star}$. Making use of this important distinction, we obtain $A_{C P}=\left[A_{\mathrm{rec}}^{\operatorname{cor}}\left(\cos \theta^{\star}\right)+A_{\mathrm{rec}}^{\operatorname{cor}}\left(-\cos \theta^{\star}\right)\right] / 2$ and $A_{\mathrm{FB}}=\left[A_{\mathrm{rec}}^{\mathrm{cor}}\left(\cos \theta^{\star}\right)-A_{\mathrm{rec}}^{\mathrm{cor}}\left(-\cos \theta^{\star}\right)\right] / 2$.

Based on a total signal yield of $34,460 \pm 273 D^{0} \rightarrow \pi^{0} \pi^{0}$ events, we obtain $A_{C P}=[-0.03 \pm 0.64$ (stat) \pm 0.10 (syst) $] \%$, which improves over the previous results [15] by an order of magnitude and shows no hint for $C P$ violation. We also measure $A_{C P}\left(D^{0} \rightarrow K_{S}^{0} \pi^{0}\right)=[-0.21 \pm 0.16$ (stat) \pm 0.07 (syst) $] \%$, which supersedes Belle's earlier result [17]. After subtracting $C P$ violation due to $K^{0}-\bar{K}^{0}$ mixing, the $C P$ asymmetry for $D^{0} \rightarrow \bar{K}^{0} \pi^{0}$ is found to be $(+0.12 \pm 0.16 \pm 0.07) \%$, again consistent with no $C P$ violation.

\section{Lepton forward-backward asymmetry in $B \rightarrow X_{S} \ell^{+} \ell^{-}$}

The flavor-changing neutral current (FCNC) transition $b \rightarrow s \ell^{+} \ell^{-}(\ell=$ $e, \mu)$ is forbidden at tree level in the SM. However, it can occur at higher order via electroweak loop (penguin) and $W^{+} W^{-}$box diagrams. The corresponding decay amplitudes are expressed in terms of the effective Wilson coefficients [19]: $C_{7}$ for the electromagnetic penguin, $C_{9}$ and $C_{10}$ for the vector and axial-vector electroweak contributions, respectively [20]. In presence of NP

contributions, these coefficients are expected to differ from SM predictions, leading to a dramatic change in the decay rate and angular distributions of 
the $b \rightarrow s \ell^{+} \ell^{-}$transition [21]. For instance, the lepton forward-backward asymmetry in the $B \rightarrow X_{S} \ell^{+} \ell^{-}$decays,

$$
A_{\mathrm{FB}}=\frac{\Gamma\left(B \rightarrow X_{S} \ell^{+} \ell^{-} ; \cos \theta>0\right)-\Gamma\left(B \rightarrow X_{S} \ell^{+} \ell^{-} ; \cos \theta<0\right)}{\Gamma\left(B \rightarrow X_{S} \ell^{+} \ell^{-} ; \cos \theta>0\right)+\Gamma\left(B \rightarrow X_{S} \ell^{+} \ell^{-} ; \cos \theta<0\right)},
$$

where $\theta$ is the angle between the $\ell^{+}\left(\ell^{-}\right)$and the $B$ meson momentum in the $\ell^{+} \ell^{-}$CM frame and $X_{S}$ is a hadronic system containing an $s$ quark, exhibits an excellent sensitivity for physics beyond the SM.

Motivated by this, we perform the first measurement of $A_{\mathrm{FB}}$ in the inclusive $B \rightarrow X_{S} \ell^{+} \ell^{-}$decays as a function of the dilepton invariant mass squared $q^{2}=m_{\ell^{+} \ell^{-}}^{2}$ [22], using $772 \times 10^{6} B \bar{B}$ pairs collected at the $\Upsilon(4 S)$ resonance. By inclusive here one means a sum of several exclusive hadronic final states representing the $X_{S}$ system. We reconstruct $B$ mesons in 18 hadronic final states with $X_{S} \equiv\{K\}\{n \pi\}, K=K^{ \pm}, K_{S}^{0}$ and $n=1 \ldots 4$ of which at most one pion can be neutral, together with two oppositely charged leptons (electrons or muons). In case of $B^{0}\left(\bar{B}^{0}\right)$ decays, only self-tagging modes with a $K^{+}\left(K^{-}\right)$are utilized. Signal events are identified with two kinematic variables, calculated in the $\Upsilon(4 S)$ rest frame: the beam-energy constrained mass $M_{\mathrm{bc}}=\sqrt{E_{\mathrm{beam}}^{2}-\left|\vec{p}_{B}\right|^{2}}$ and the energy difference $\Delta E=E_{B}-E_{\mathrm{beam}}$, where $E_{\text {beam }}$ is the beam energy, and $\left(E_{B}, \vec{p}_{B}\right)$ are the reconstructed energy and momentum of the $B$-meson candidate. To reduce contamination from the $e^{+} e^{-} \rightarrow q \bar{q}(q=u, d, s, c)$ continuum background, we use a neural network mostly based event topology and $B$ vertex-fit quality. Charmonia contributions from $B \rightarrow X_{S} J / \psi[\psi(2 S)]$ with $J / \psi[\psi(2 S)] \rightarrow \ell^{+} \ell^{-}$, are suppressed by rejecting ("vetoing") events with a dilepton invariant mass in the following two ranges: -400 to $150 \mathrm{MeV} / \mathrm{c}^{2}$ (-250 to $100 \mathrm{MeV} / \mathrm{c}^{2}$ ) and -250 to $100 \mathrm{MeV} / \mathrm{c}^{2}$ (-150 to $100 \mathrm{MeV} / \mathrm{c}^{2}$ ) around the nominal $\mathrm{J} / \psi$ and $\psi(2 S)$ mass [6] mass for the electron (muon) channel.

In total, $140 \pm 19$ (stat) $B \rightarrow X_{S} e^{-} e^{+}$and $161 \pm 20$ (stat) $B \rightarrow X_{S} \mu^{+} \mu^{-}$ signal candidates are selected in the data sample. To study the $q^{2}$ dependence of $A_{\mathrm{FB}}$, we divide the data into four $q^{2}$ bins: [0.2, 4.3], [4.3,7.3(8.1)], $[10.5(10.2), 11.8(12.5)]$, and $[14.3,25.0] \mathrm{GeV}^{2} / c^{4}$ for the electron (muon) channel, where the gap regions correspond to the veto described earlier. The $A_{\mathrm{FB}}$ value is found to be consistent with the SM prediction in the two intermediate $q^{2}$ bins, while it deviates from the SM in the lowest $q^{2}$ bin by $1.8 \sigma$ (including the systematic uncertainty). Results in the last two bins exclude $A_{\mathrm{FB}}<0$ at a $2.3 \sigma$ level. 


\section{Observation of the decay $B^{0} \rightarrow \eta^{\prime} K^{\star}(892)^{0}$}

The $B^{0} \rightarrow \eta^{\prime} K^{\star}(892)^{0}$ decay proceeds via the $b \rightarrow s$ penguin and Cabibbosuppressed $b \rightarrow u$ tree diagrams. There is a destructive interference between two contributing amplitudes leading to a small decay branching fraction. The interference pattern could also give rise to a large direct $C P$ violation. Typical branching fraction values calculated within the framework of perturbative QCD [23], QCD factorization [24], soft collinear effective theory [25] and $S U(3)$ flavor symmetry [26] are in the range 1.2-6.3\%. In the past, Belle [27] and BABAR [28] have searched for $B^{0} \rightarrow \eta^{\prime} K^{\star}(892)^{0}$ with the latter reporting the first evidence at a $4 \sigma$ level. So far, $C P$ violation has not been probed in the decay.

We search for $B^{0} \rightarrow \eta^{\prime} K^{\star}(892)^{0}$ [29] using a data sample of $772 \times 10^{6}$ $B \bar{B}$ pairs recorded at the $\Upsilon(4 S)$ resonance. The decay candidates are reconstructed from the subsequent decay modes $\eta^{\prime} \rightarrow \eta \pi^{+} \pi^{-}, \eta \rightarrow \gamma \gamma$ and $K^{\star}(892)^{0} \rightarrow K^{+} \pi^{-}$. Based on an extended maximum likelihood fit to their distributions of $M_{\mathrm{bc}}, \Delta E$, continuum suppression variable, and the cosine of the $K^{\star}$ helicity angle, we extract a signal yield of $31 \pm 9$ events with a significance of $5 \sigma$, including systematic uncertainties. This constitutes the first observation of the decay channel. The yield is translated to a branching fraction $\mathcal{B}\left[B^{0} \rightarrow \eta^{\prime} K^{\star}(892)^{0}\right]=[2.6 \pm 0.7$ (stat) \pm 0.2 (syst) $] \times 10^{-6}$, in a good agreement with theory predictions. We also measure direct $C P$ violation by splitting the obtained yield according to the flavor of the $B$ meson, based on the sign of the daughter kaon from the $K^{\star}$ decay. The obtained result, $A_{C P}=-0.22 \pm 0.29$ (stat)0.07(syst), is consistent with no $C P$ violation.

\section{An amplitude analysis of $B \rightarrow J / \psi K \pi$}

Recently, a number of new charmonium-like states have been observed at the $B$ factories and elsewhere. Some of them especially the charged ones look very much like exotic, defying predictions of the quark model. The first one in the series, the $Z_{c}(4430)^{+}$, was discovered by Belle in the $\psi(2 S) \pi^{+}$invariant mass spectrum in $B^{0} \rightarrow \psi(2 S) K^{-} \pi^{+}$[30], followed by two more states, the $Z_{c}(4050)^{+}$and $Z_{c}(4250)^{+}$, in $B^{0} \rightarrow \chi_{c 1} K^{-} \pi^{+}$decays [31]. Lately, BESIII has joined the game by observing $Z_{c}(3900)^{+}$in the $J / \psi \pi^{+}$invariant mass spectrum in $Y(4260) \rightarrow J / \psi \pi^{+} \pi^{-}$[32]. In a back-to-back publication [33], Belle corroborated the finding. 
Motivated by these exciting results, we perform an amplitude analysis [35] of the decay $B^{0} \rightarrow J / \psi K^{-} \pi^{+}$, with $J / \psi \rightarrow \mu^{+} \mu^{-}$or $e^{+} e^{-}$, using a data sample of $772 \times 10^{6} B \bar{B}$ pairs. Our analysis strategy is similar to a recent study of $B^{0} \rightarrow \psi(2 S) K^{-} \pi^{+}$34]. In addition to the known $Z_{c}(4430)^{+}$state, we find a new charmonium-like state $Z_{c}(4200)^{+}$in the $J / \psi \pi^{+}$invariant mass spectrum with a significance exceeding $6 \sigma$. The minimal quark content of this state is exotic: $\mid c \bar{c} u \bar{d}>$. Its mass and decay width are measured to be $\left[4196_{-29}^{+31}(\text { stat })_{-13}^{+17}(\right.$ syst $\left.)\right] \mathrm{MeV} / c^{2}$ and $[370 \pm 70 \text { (stat })_{-132}^{+70}$ (syst) $] \mathrm{MeV}$, respectively. The preferred spin-parity quantum numbers are $J^{P}=1^{+}$.

\section{Summary and outlook}

Though close to five years have passed by since its data taking, Belle continues to produce high quality results. A small sample of those based on the full statistics are presented here. That includes: a) first observation of $D^{0}$ - $\bar{D}^{0}$ mixing using $D \rightarrow K \pi$ decays in $e^{+} e^{-}$collisions, b) a $2.5 \sigma$ evidence for charm mixing and no hint for $C P$ violation in $D^{0} \rightarrow K_{S}^{0} \pi^{+} \pi^{-}$, c) an order-ofmagnitude improvement over the previous results for $A_{C P}$ in the decay $D^{0} \rightarrow$ $\pi^{0} \pi^{0}$, d) a $1.8 \sigma$ difference with respect to the SM prediction in the lepton forward-backward asymmetry at low $q^{2}$ in the inclusive $B \rightarrow X_{S} \ell^{+} \ell^{-}$decays,

e) first observation of the charmless hadronic decay $B^{0} \rightarrow \eta^{\prime} K^{\star}(892)^{0}$, and f) observation of a new charged charmonium-like state in $B^{0} \rightarrow J / \psi K^{-} \pi^{+}$. Such kind of unique explorations at the next-generation $e^{+} e^{-}$flavor factory will continue with the upcoming Belle II experiment [36].

[1] N. Cabibbo, Phys. Rev. Lett. 10, 531 (1963); M. Kobayashi and T. Maskawa, Prog. Theor. Phys. 49, 652 (1973).

[2] S. Glashow, J. Illiopoulos, and L. Maiani, Phys. Rev. D 2, 1285 (1970).

[3] I. I. Bigi and N. G. Uraltsev, Nucl. Phys. B592, 92 (2001); A. A. Petrov, Nucl. Phys. Proc. Suppl. 142, 333 (2005).

[4] Y. Grossman, A. L. Kagan, and Y. Nir, Phys. Rev. D 75, 036008 (2007).

[5] B. R. Ko et al. (Belle Collaboration), Phys. Rev. Lett. 112, 111801 (2014).

[6] K. A. Olive et al. (Particle Data Group), Chi. Phys. C 38, 090001 (2014). 
[7] R. Aaij et al. (LHCb Collaboration), Phys. Rev. Lett. 110, 101802 (2013); T. Aaltonen et al. (CDF Collaboration), Phys. Rev. Lett. 111, 231802 (2013); R. Aaij et al. (LHCb Collaboration), Phys. Rev. Lett. 111, 251801 (2013).

[8] T. Peng et al. (Belle Collaboration), Phys. Rev. D 89, 091103 (2014).

[9] Chapter 13 of The Physics of the B Factories, Ed. A. J. Bevan, B. Golob, Th. Mannel, S. Prell, and B. D. Yabsley, submitted to EPJC, arXiv:1406.6311 (2014).

[10] H.-Y. Cheng and C.W. Chiang, Phys. Rev. D 85, 034036 (2012); 85, 079903(E) (2012); 86, 014014 (2012).

[11] G. Hiller, M. Jung, and S. Schacht, Phys. Rev. D 87, 014014 (2013).

[12] R. Aaij et al. (LHCb Collaboration), Phys. Rev. Lett. 108, 111602 (2012).

[13] T. Aaltonen et al. (CDF Collaboration), Phys. Rev. Lett. 109, 111801 (2012).

[14] B. Bhattacharya, M. Gronau, and J. L. Rosner Phys. Rev. D 85, 054014 (2012).

[15] G. Bonvicini et al. (CLEO Collaboration), Phys. Rev. D 63, 071101 (2001).

[16] N. K. Nisar et al. (Belle Collaboration), Phys. Rev. Lett. 112, 211601 (2014).

[17] B. R. Ko et al. (Belle Collaboration), Phys. Rev. Lett. 106, 211801 (2011).

[18] Y. Amhis et al. (Heavy Flavor Averaging Group), arXiv:1207.1158 (2012); updates available at http://www.slac.stanford.edu/xorg/hfag.

[19] K. G. Wilson, Phys. Rev. 179, 1499 (1969).

[20] G. Buchalla, A. J. Buras, and M. E. Lautenbacher, Rev. Mod. Phys. 68, 1125 (1996). 
[21] A. Ali, E. Lunghi, C. Greub, and G. Hiller, Phys. Rev. D 66, 034002 (2002).

[22] Y. Sato et al. (Belle Collaboration), arXiv:1402.7134 (2014).

[23] A. G. Akeroyd, C. H. Chen, and C. Q. Geng, Phys. Rev. D 75, 054003 (2007).

[24] M. Beneke and M. Neubert, Nucl. Phys. B 675, 333 (2003).

[25] W. Wang, Y. M. Wang, D. S. Yang, and C. D. Lu, Phys. Rev. D 78, 034011 (2008).

[26] C.-W. Chiang, M. Gronau, Z. Luo, J. L. Rosner, and D. A. Suprun, Phys. Rev. D 69, 034001 (2004).

[27] J. Schümann et al. (Belle Collaboration), Phys. Rev. D 75, 092002 (2007).

[28] P. del Amo Sanchez et al. (BABAR Collaboration), Phys. Rev. D 82, 011502 (2010).

[29] S. Sato et al. (Belle Collaboration), Phys. Rev. D 90, 072009 (2014).

[30] S. K. Choi et al. (Belle Collaboration), Phys. Rev. Lett. 100, 142001 (2008).

[31] R. Mizuk et al. (Belle Collaboration), Phys. Rev. D 78, 072004 (2008).

[32] M. Ablikim et al. (BESIII Collaboration), Phys. Rev. Lett. 110, 252001 (2013).

[33] Z. Q. Liu et al. (Belle Collaboration), Phys. Rev. Lett. 110, 252002 (2013).

[34] K. Chilikin et al. (Belle Collaboration), Phys. Rev. D 88, 074026 (2013).

[35] K. Chilikin et al. (Belle Collaboration), arXiv:1408.6457 (2014).

[36] P. Urquijo, these proceedings. 